\title{
FAMILY OF GENERALIZED TRIADIC KOCH FRACTALS: DIMENTIONS AND FOURIER IMAGES
}

\author{
${ }^{1,2}$ Galina V. Arzamastseva, ${ }^{1}$ Mikhail G. Evtikhov, ${ }^{1}$ Feodor V. Lisovsky, ${ }^{1}$ Ekaterina G. \\ Mansvetova \\ ${ }^{1}$ Kotel'nikov Institute of Radio-Engineering and Electronics, Fryazino Branch, Russian Academy of Science, \\ http:/ / fire.relarn.ru \\ 141120 Fryazino, Moscow region, Russian Federation \\ lisf@df.ru, arzamastseva@mail.ru,emg20022002@mail.ru,mansvetova_eg@mail.ru \\ ${ }^{2}$ Modern Humanitarian Academy, http://www.muh.ru \\ 109029 Moscow, Russian Federation
}

\begin{abstract}
Fourier images of generalized triadic Koch fractals (curves and snowflakes) with variable vertex angle of generator were obtained by digital methods. The comparison of different methods of fractal dimensions determination using Fraunhofer diffraction patterns was made. Analysis of the size ratio dependence of central (fractal) and peripheral (lattice) parts of diffraction pattern both upon vertex angle at a fixed value of prefractal generation number and upon prefractal generation number at a fixed value of vertex angle was made. The features of Koch curves and Koch snowflakes Fourier images are discussed.

Keywords: annular zones method, box-counting method, digital methods, Fourier image, fractal dimension, Fraunhofer diffraction, Koch curve, Koch fractal, Koch snowflake, method of circles, scaling factor, scaling invariance, symmetry.
\end{abstract}

UDC 51.74; 535.42

Bibliography - 27 references

Recieved 17.11.2015

RENSIT, 8(1):81-90

DOI: $10.17725 /$ rensit.2016.08.081

\section{ConTENTS:}

1. INTRODUCTION (81)

2. Declaration of procedure for Koch FRACTALS Fourier IMAGES ObTAINING (82)

3. Methods of Koch fractals dimension DETERMINATION (83)

4. Fourier images of Koch Curves with VARIABLE VERTEX ANGLE OF GENERATOR (83)

5. Dependence of Koch Curves dimension ON VERTEX ANGLE OF GENERATOR (84)

6. Properties of Koch curves Fourier IMAGES (85)

7. Properties of Koch snowflakes Fourier IMAGES (87)

8. Properties of nodified Koch curves Fourier IMAGES (88)

9. ConClusion (88)

REFERENCIES (89)

\section{INTRODUCTION}

Externally similar to the snowflake outline fractal (grandparent of fractals family which will be discussed below) was named in honor of Swedish mathematician Helge von Koch, who first described it in published in 1904 year article the title of which in French sounds like "Of nontangent continuous curve to obtain an elementary geometric constructions" [1].

The Koch fractals were quite a complicated genealogy. It turned out that a year before the Japanese mathematician Takagi Teiji published an article with almost the identical title "Simple example of continuous function without derivatives" [2], where he described the socalled "blancmange" curve (currently referred to as Takagi-Landsberg curve), which was the nearest relative of the Koch snowflake. Then the family of curves under consideration was expanded in 1933 by Hildebrandt [3] and in 1957 
by de Rham [4]. The last extension was the most representative: the individual cases of curves deFrame are such wellknown classical fractals like the Koch snowflake, spacefilling Peano curves, curves of Cesaro-Faber, Takagi-Landsberg, Levi, etc., see [5].

In the present work we studied the properties obtained by numerical methods Fourier images and Hausdorff dimension spectra of the triad geometric fractals family with generator in the form of a symmetrical four-segment broken line with an arbitrary angle between the central units and with the initiator (broken line) in the form of a straight line (Koch curve) or in the form of an equilateral triangle (Koch snowflake). When constructing a fractal, at each step the segments of the initial broken line are divided symmetrically relative to the center into three equal (for $\alpha=60^{\circ}$ ) or unequal (for $\alpha \neq 60^{\circ}$ ) parts (hence "triad" in the name of a family).

Used in the present work, the term "Fourier image" actually refers to the 2D-graphic representation of distribution of the squared modules of the component of the Fourier transform for images of fractals (see figures in text). The resulting picture is close to that obtained by Fraunhofer diffraction of electromagnetic waves on fractals, which makes possible to run verification field experiments.

\section{DECLARATION OF PROCEDURE FOR KOCH FRACTALS FOURIER IMAGES OBTAINING}

Used in the present work a procedure for determination of fractal object picture Fourier images (i.e., diffraction patterns in the Fraunhofer zone) using numerical methods, previously described and applied to study the fractallike domain structures in magnetic films [6], later was applied to the real test objects (mono- and biperiodical domain structures), when it was possible in the visible range of wavelengths to observe directly and photograph the diffraction pattern by the "transmission" mode. Fourier images, obtained by digitized pictures of domain structures, correspond both the results of the theoretical calculation and the experimentally observed diffraction patterns [7]. In addition, similar numerical methods have been used for a number of other fractal objects [8], for which the phenomenon of diffraction have been well studied by other authors [9-11]. The agreement obtained Fourier images (diffraction patterns) with the results of these experimental and theoretical studies was quite good. Note that numerical methods are quite simple and do not require an analytical description of fractals, that was used, for example, the authors of the works $[12,13]$.

The first step in the implementation of used numerical methods was to generate black-andwhite raster image of selected fractal objects with the help of specially designed programs. Further images of fractals were approximated by a grid function on a uniform grid with number of nodes $n_{1} \times n_{2}$, where the values $n_{1}$ и $n_{2}$ were chosen sufficiently large (up to 4096) in order to ensure adequate approximation of the smallest details and have the opportunity to explore generation of prefractals with high numbers of generations. For digitized pictures using fast Fourier transform we determined the values of the Fourier component squared modules, i.e. the spectral distribution of intensity $I$ of diffracted radiation in the zone of Fraunhofer. To display the intensity $I$ of the diffraction maxima on the 2D-plane, one can use either a linear (or logarithmic) scale intensity using different gray levels, or representation of $I$ values in the form of circles with radius proportional to intensity (or logarithm of it), where coefficient of proportionality is chosen for reasons of optimal illustrativity of images. This article used the second method, modified by additional Gaussian blur of circles displaying the values of $I$.

In the following sections, according to made in the introduction comments of, 
we'll use for Koch fractal with the generator in the form of symmetrical relative to the middle fourbar broken line with an arbitrary angle between the central bars name "Koch curve", if the initiator is a line segment, and the name "Koch snowflake", if the initiator is an equilateral triangle (see Fig. 1). Note that to preserve the self-similarity of prefractal by iterations all the segments of generator in a form of broken line must have the same length. When $\alpha \rightarrow 0^{\circ}$, the broken line becomes delta-shaped, when $\alpha \rightarrow 180^{\circ}$, it merges with a initiator line; case $\alpha=60^{\circ}$ corresponds to the classical Koch curve.

\section{METHODS OF KOCH FRACTALS DIMENSION DETERMINATION}

Analysis of the diffraction pattern allows to determine the Hausdorff dimension of fractals studied by two different numerical methods. The first method (hereinafter, method of circles) based on a numerical determination of the average resultant intensity of the diffracted radiation

$$
\bar{I}\left(r_{k}\right)=\frac{1}{\pi r_{k}^{2}} \int_{0}^{2 \pi} \int_{0}^{r_{k}} I(\rho, \varphi) \rho d \rho d \varphi
$$

in the circles of variable radius $r_{k}=r_{0}+k \delta_{\mathrm{r}}$ centered at the position of the main diffraction maximum ( $\varrho$ and $\varphi-$ polar coordinates in the plane of diffraction, $r_{0}$ and $\delta_{\mathrm{r}}-$ are the initial radius and increment of radius as well $k=0,1$, $2, \ldots)$ and then using the formula

$a$

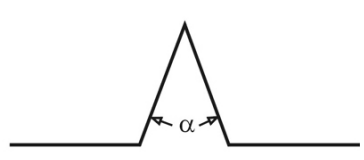

$b$

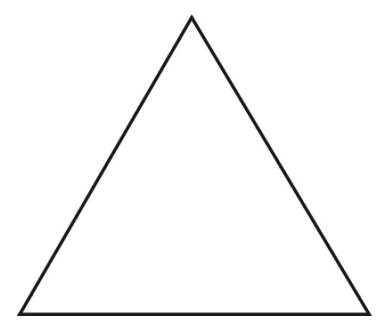

Fig. 1. Initiator and generator of the triad generalized Koch curves (a) and Koch snowflakes (b).
$\bar{I}\left(r_{k}\right)=I_{0} e^{-D_{f}}$,

from which it follows that the value of fractal dimension $D_{f}$ equals the modulo of the angular coefficient of the straight line, approximating the dependence $\bar{I}\left(r_{k}\right)$ in double logarithmic scale.

In the second method (hereinafter, method of the annular zones) the diffraction plane is divided into annular regions by concentric system of circles with radii $r_{k}=r_{0} m^{k}$, where $r_{0}-$ is an initial radius and $m>1$ is a constant multiplier. Then the average resulting intensity of the diffracted radiation inside of each ring is calculated

$$
\bar{I}\left(r_{k}\right)=\frac{1}{\pi m^{2 k} r_{0}^{2}\left(m^{2}-1\right)} \int_{0}^{2 \pi} \int_{m^{k} r_{0}}^{m^{k+1} r_{0}} I(\rho, \varphi) \rho d \rho d \varphi,
$$

after that the dependence $\bar{I}\left(r_{k}\right)$ in double logarithmic scale is built, and module of the slope of the linear section of which determines the fractal dimension $D_{f}$. For self-similar fractals, to discussed in this work fractals belong, the value of coincides with the scaling factor of the elements in the transition between adjacent hierarchical levels of the fractal, and the value of $r_{0}$ must be chosen so that each of the annular zones contained only congruent fragments of the dif-fraction patterns [9-13].

The dimension of the fractals can be determined also by nonspectral box counting technique, which uses a built in double logarithmic scale the dependence $N\left(\varepsilon_{k}\right)=f(1 /$ $\left.\varepsilon_{k}\right)$, where $N\left(\varepsilon_{k}\right)$ - needed to fully cover the fractal is the number of squares with side length $\varepsilon_{k} \underset{k \rightarrow \infty}{\rightarrow} 0$ [14-19]. The value of dimension $D_{f}$ is determined with an angular coefficient of dependence $N\left(\varepsilon_{k}\right)$.

\section{FOURIER IMAGES OF KOCH CURVES WITH VARIABLE VERTEX ANGLE OF GENERATOR}

In Fig. 2 and Fig. 3 the Fourier images (diffraction pattern) of Koch curves with different values of the angle $\alpha$ (values are given under each figure) obtained by described in 
section 2 method are shown. The initiator for all fractals served line segment with length of 4000 pixels. The pictures of corresponding 3 -rd generation curves are placed on top of each figure for clarity; to obtain Fourier images prefractals of higher generation were used. The diffraction pattern Fig. 2a-g belong to prefractal of the 6-th generation of the diffraction pattern Fig. $2 \mathrm{~h}$ and Fig. 3a-d - to prefractal of the 5-th generation of the diffraction pattern Fig. 3e-h to prefractals of 4-th generation.

The diffraction pattern for each value of the angle $\alpha$ is given in two scales, differ in two times: at the top - smaller, at the bottom larger, allowing one to examine the details of the center of the diffraction pattern corresponding to the fractal part of spectrum. Fig. 2a-h and Fig. 3a-d have the same image scale in each row (top or bottom); in Fig. 3e-h we used two-fold increase, since the reduction of the number of the prefractals generation
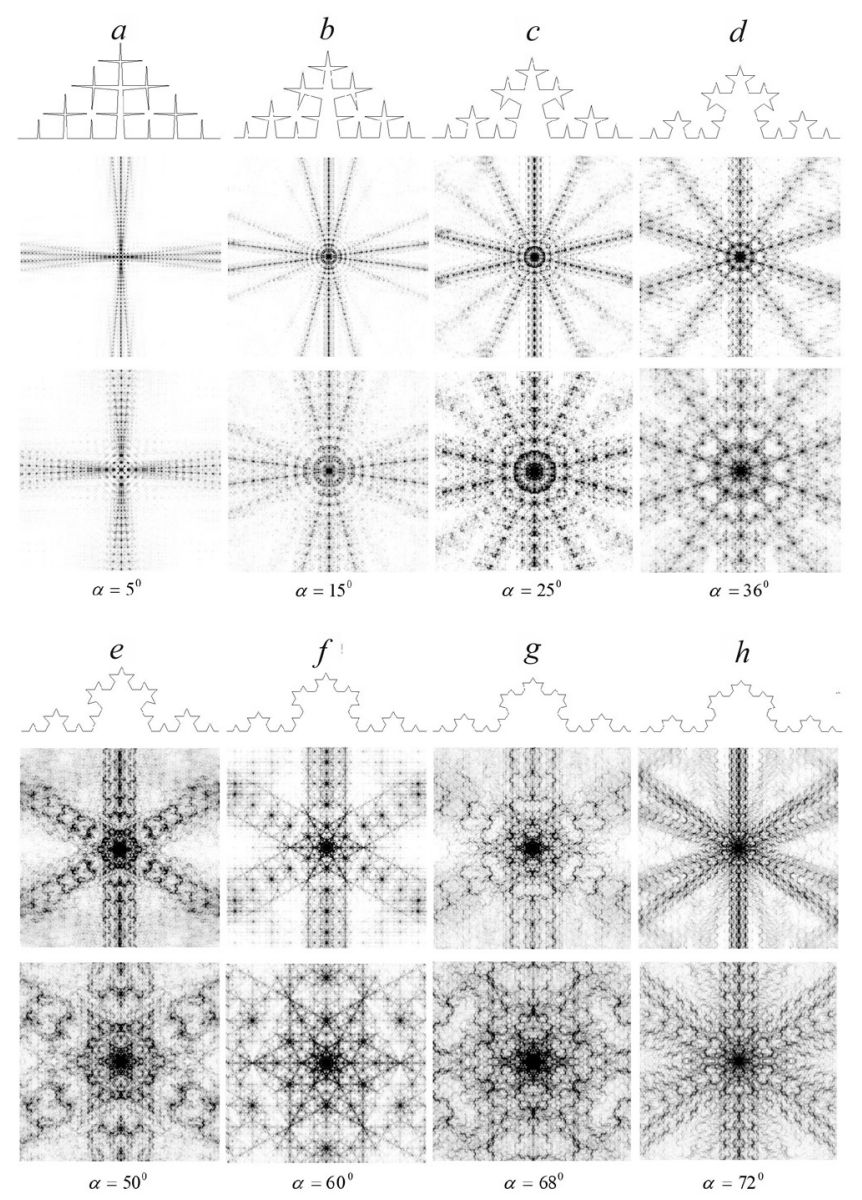

Fig. 2. Fourier images of Koch curves with different values of the vertex generator angle in the in-terval from $\alpha=2^{\circ}$ to $72^{\circ}$. accompanied by a strong decrease in the size of the central (fractal) part (see details later in section 6).

Comparison of diffraction patterns Fig. 2 and Fig. 3 with the same image scale and the same prefractals generations, differing only angle $\alpha$ value, shows that the fractal part of the Fourier images is narrowed by decreasing this angle throughout the studied interval changes. Especially strongly this effect is manifested if $\alpha \rightarrow 0^{\circ}$, when the spectrum is dominated lattice (associated with the ordering) part (Fig. 2a). The dimension of the fractal in this case tends to 2 (see below), i.e., it acquires the features of a space-filling curve.

\section{DEPENDENCE OF KOCH CURVES DIMENSION ON VERTEX ANGLE OF GENERATOR}

A diffractograms similar to that shown in Fig. 2 and Fig. 3, were used to determine the dimensions of Koch curves with different values of the vertex angle of the generator
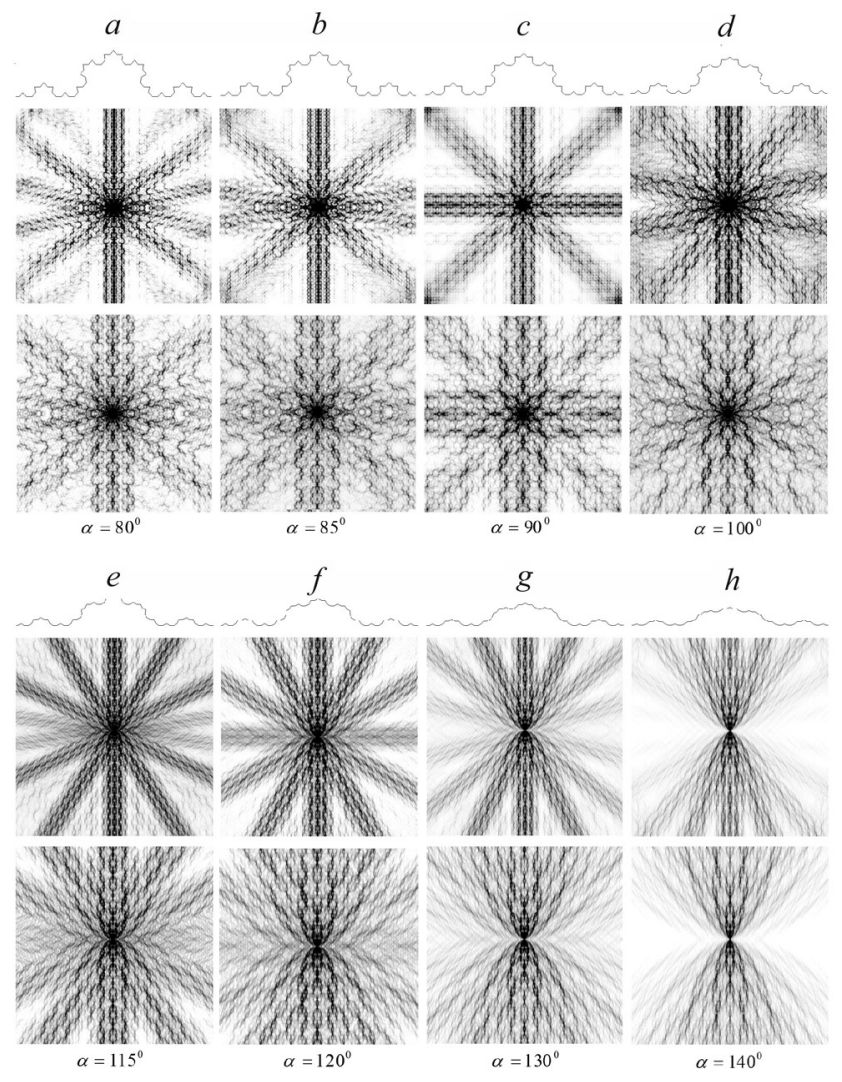

Fig. 3. Fourier images of Koch curves with different values of the vertex generator angle in the in-terval from $80^{\circ}$ to $140^{\circ}$. 
using method of circles and of annular zones. Attempts to use box counting method showed that a reliable allocation of the linear section of the curve according to the considered family of fractals (especially for $\alpha<8^{\circ}$ ) is associated with significant difficulties, therefore this method was abandoned.

Graphs of the dependences $\bar{I}\left(r_{k}\right)$ in doublelogarithmic scale are shown in Fig. 4 for the method of annular zones (Fig. $4 a-c$ ) and the method of circles (Fig. $4 d-f$ ) at various values of the angle $\alpha$. The dashed lines in Fig. 4 have a slope equal to the theoretical value of the dimension $D_{f}$ taken with the opposite sign, which is determined by the expression $[14,18$, 20]

$$
D_{f}=\frac{2 \log 2}{\log 2+\log [1+\sin (\alpha / 2)]}=\frac{2 \log 2}{\log m},
$$

where $m=2[1+\sin (\alpha / 2)]$ is a scale factor. It is seen that if the dependence $\bar{I}\left(r_{k}\right)$ for method of circles in almost all the range of changes
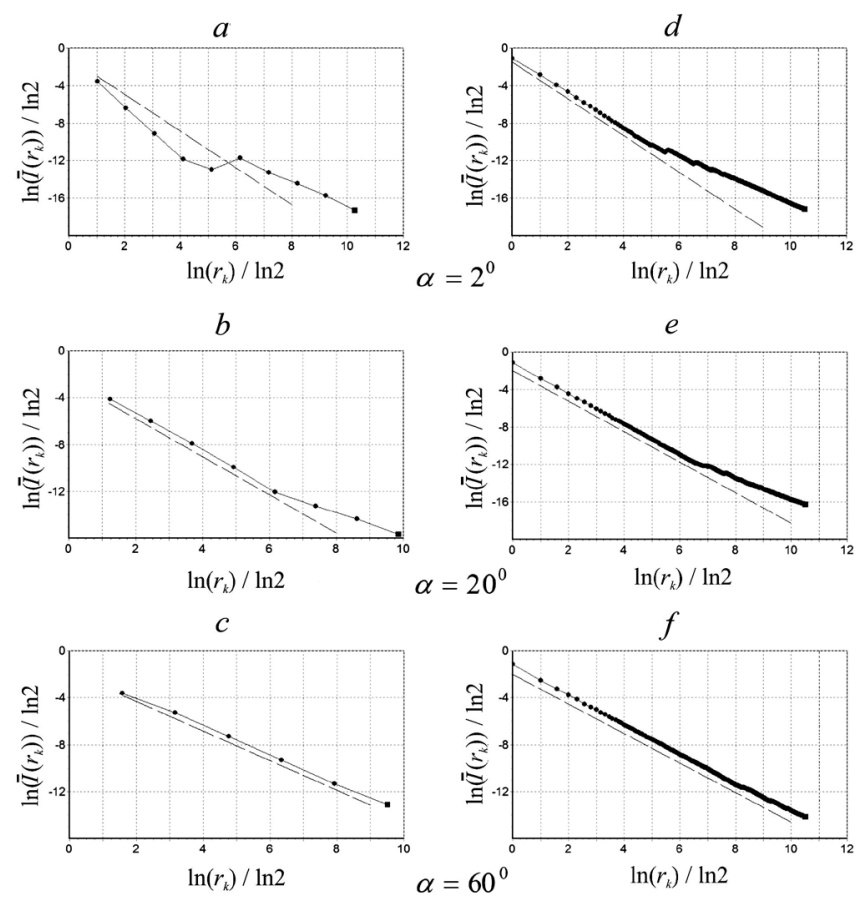

Fig. 4. Graphs of the dependences $\bar{I}\left(r_{k}\right)$ in double-logarithmic scale for the method of the annular zones $(a, b, c)$ and the method of the circles $(d, e, f)$ for different values of the angle $\alpha$. The slope of the dashed lines corresponds to the theoretical value of $D_{f}$ $\alpha$ has linear part sufficient to determine value of $D_{f}$ (Fig. $\left.4 d-f\right)$, for method of annular zones linear part appears only when $\alpha \geq 7.5^{\circ}$. With increasing of $\alpha$ linearity region for dependence $\bar{I}\left(r_{k}\right)$ for both methods expands. Theoretical dependence of fractal dimension on angle $\beta$ $=90^{\circ}-\alpha / 2$ is shown in Fig. 5, where one can see also the corresponding "experimental" values of fractal dimension $D_{f}$ obtained by method of circles (O) and method of annular zones $(X)$.

If $\alpha$ is approaching to $180^{\circ}$, the scale factor $m \rightarrow 4$, and the dimension $D_{f} \rightarrow 1$, i.e. the fractal curve degenerates into a line segment. In another limiting case, when the scaling factor $m \rightarrow 2$ and the dimension $D_{f} \rightarrow 2$, i.e. the fractal curve tends to fill the entire plane, and therefore, as was shown by us earlier for Peano and Gosper curves, method of the annular zones failes, as the lattice portion of the diffraction pattern begins to dominate the fractal due to the high degree of ordering space-filling curves [21].

\section{PROPERTIES OF KOCH CURVES FOURIER IMAGES}

Received by numerical methods Fourier images of Koch curves with $\alpha=2^{\circ}$ of the 5-th, 6-th

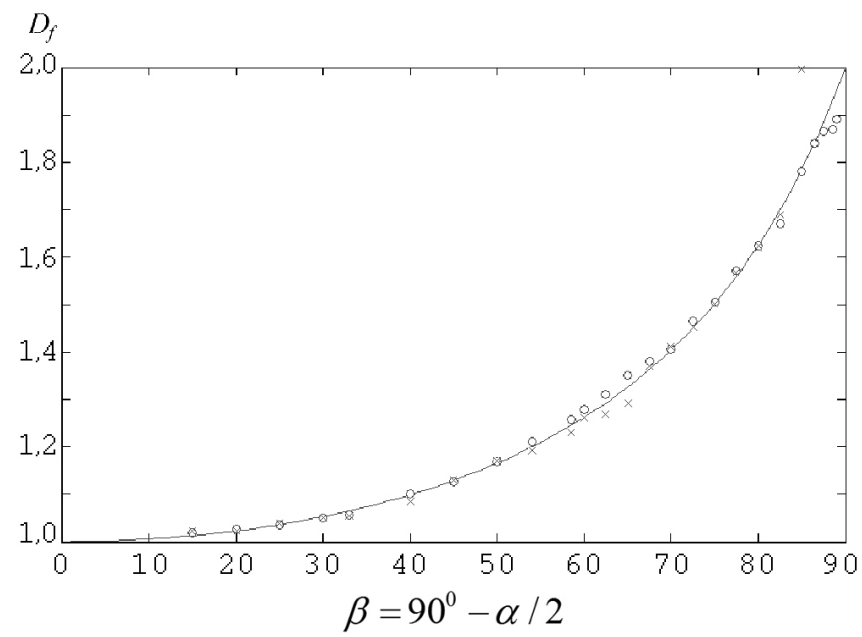

Fig. 5. The theoretical dependence of fractal dimension $D_{f}$ on the angle $\beta=90^{\circ}-\alpha / 2$ and the corresponding "experimental" values obtained by the method of circles (O) and the method of an-nular zones $(X)$. 
and 7-th generation respectively are shown in Fig. 6a-c, theoretical (dashed line) and calculated by the method of ring zones (solid lines) dependencies $\bar{I}\left(r_{k}\right)$ are given in Fig. 6d-f. It is seen that although the central part of the diffraction pattern is expanded with increase of number of the prefractal generation order, the essential approaching of curve to the theoretical one is absent, and even to the 7-th generation (Fig. 6f) selection of linear curve region is impossible.

On the basis on the analysis of investigated Koch curves Fourier images with different values of the angle $\alpha$ (see Fig. 2, 3 and 6) quantitative relationship between size of the central (fractal) part and number of prefractal gener-ation order was established. It was found that increasing generation number per unit leads to en-larging of spectrum fractal part in proportion to a scale factor $m$, that is, the
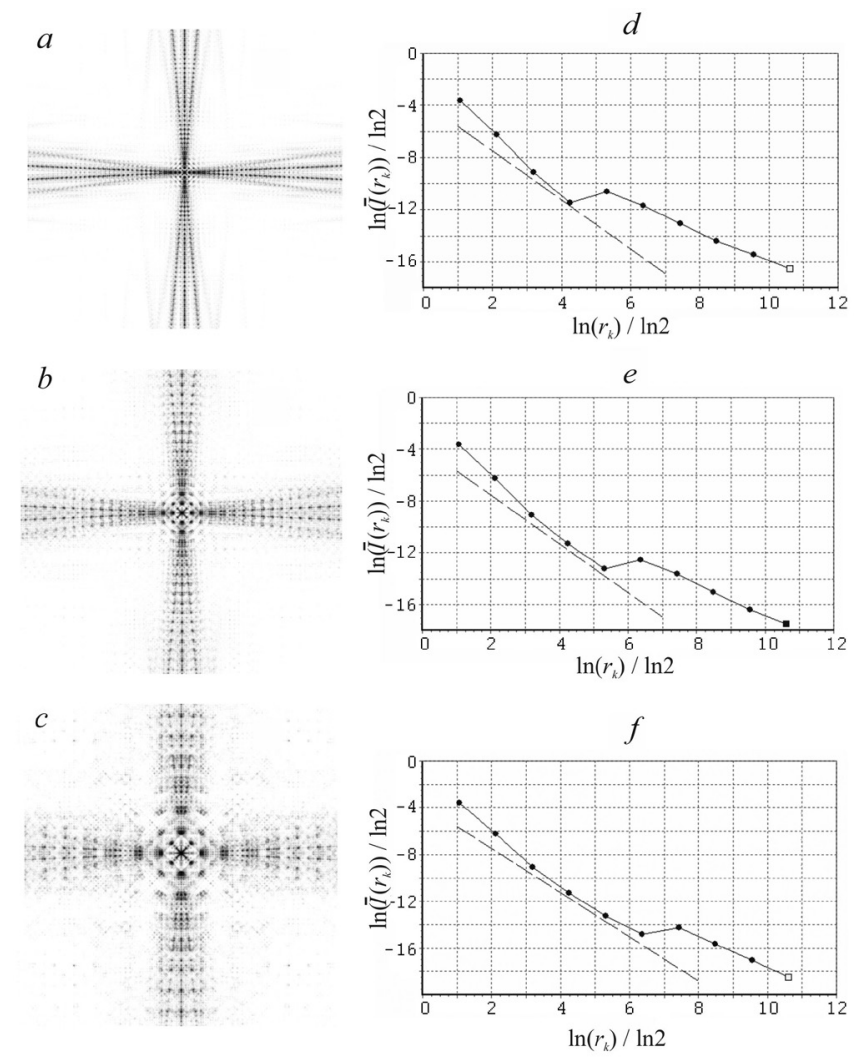

Fig. 6. Fourier-images of the Koch curve with an angle $\alpha=2^{\circ}$ for prefractals of 5-th, 6 -th and 7-th generation, respectively (a,b,c) and theoretical (dashed lines) and obtained by ring rones (solid lines) dependences $\bar{I}\left(r_{k}\right)$ for the same generations of prefractals respectively $(d, e, f)$. strongest effect occurs when $\alpha \rightarrow 180^{\circ}$ and $m$ $\rightarrow 4$. This is consistent with the results obtained in [11], where it was found that in the particular case for "classical" Koch snowflake with the angle $\alpha=60^{\circ}$ and scale factor $m=3$ linear dimensions of the fractal part increase three times while increasing prefractal generation numbers per unit.

It was also established that the fractal part of the diffraction pattern has a scale invariance in the entire studied range of angles $\alpha$, and that change of this angle leads also to significant transformation of the peripheral (lattice) part of the diffraction pattern.

General properties of the Fourier images of the Koch curve at any value of the angle are the presence of a symmetry center at the point corresponding to the zero diffraction maximum, and passing through this point of the axis of symmetry of the second order and two planes of sym-metry (vertical and horizontal). At the angles $\alpha=180^{\circ} / n$, where $n \geq 2$, in Fourier images of the ideal (infinite) fractal should appear additional axes of symmetry of the order $2 n$. This trend is quite clearly visible in Fig. 2d,f and Fig. 3c (the axis of symmetry of the 10-th, 6-th and 4-th order, respectively), but virtually absent in Fig. $2 a$ and 2 b, where should occur the axis of symmetry 72 -th and 24-th order, respectively, as to get close to the Fourier images of endless fractals by numerical methods while decreasing the angle $\alpha$, one must use increasingly higher generations of prefractal. Generally speaking, even in Fig. 2d,f and $3 c$ it is seen that upon rotation of Fourier images for the angles $36^{\circ}, 60^{\circ}$ and $90^{\circ}$, there really exists an overlay of the diffraction peaks, but their intensities differ.

Roughly the structure of all diffraction patterns can be considered as spoky-ringlike, and the "spokes" are more pronounced in peripheral (lattice) region, and the "rings" - in the central (fractal). If there are additional axes of symmetry $\left(\alpha=180^{\circ} / n\right.$, гАе $\left.n \geq 2\right)$ structure is much more complicated, at the same time greatly increases 
the clarity of the image diffraction patterns (see Fig. 2b,d,f and $3 \mathrm{c}$ ). With angle $\alpha$ increasing in the range of $25^{\circ} \leq \alpha<90^{\circ}$ the number of "spokes" monotonously decreases, and then (at $100^{\circ} \leq$ $\alpha<140^{\circ}$ ) again begins to increase due to the appearance in the Fourier images, the structure of which loses the ring component, additional "spokes" different from the mains in contrast (see Fig. 3e-h).

The clearest diffraction pattern (especially its fractal part), consisting of local maxima of dif-ferent intensity is observed for the Koch curve with an angle $\alpha=60^{\circ}$ (Fig. 2f). For all other values of the angle $\alpha$ diffraction peaks are "smeared", except the case $\alpha=36^{\circ}$, when high image definition also achieves (see Fig. 2d). The central part of the Fourier image of this fractal in doubled scale is shown in Fig. 7.

\section{PROPERTIES OF KOCH SNOWFLAKES FOURIER IMAGES}

Were also studied the properties of Fourier images of Koch snowflakes with initiator in a form of an equilateral triangle. The most symmetrical and sharp diffraction pattern for $\alpha=60^{\circ}$ almost completely coincide with the observed for the appropriate Koch curve (Fig. $2 \mathrm{f})$, however, the Fourier images of snowflakes for some values of angle $\alpha \neq 60^{\circ}$, as seen in Fig. 8, were much more sharp and had a higher degree of localization with respect to the Fourier images of Koch curves. In the left column of Fig. 8 pictures of the corresponding snowflake of 3-rd generation are shown, in central column - diffraction pattern, received for prefractal of

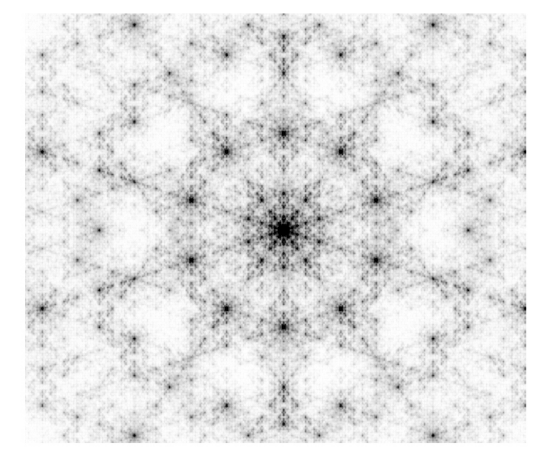

Fig. 7. The central part of the Koch curve Fourier image with an angle $\alpha=36^{\circ}$ at high magnifica-tion.

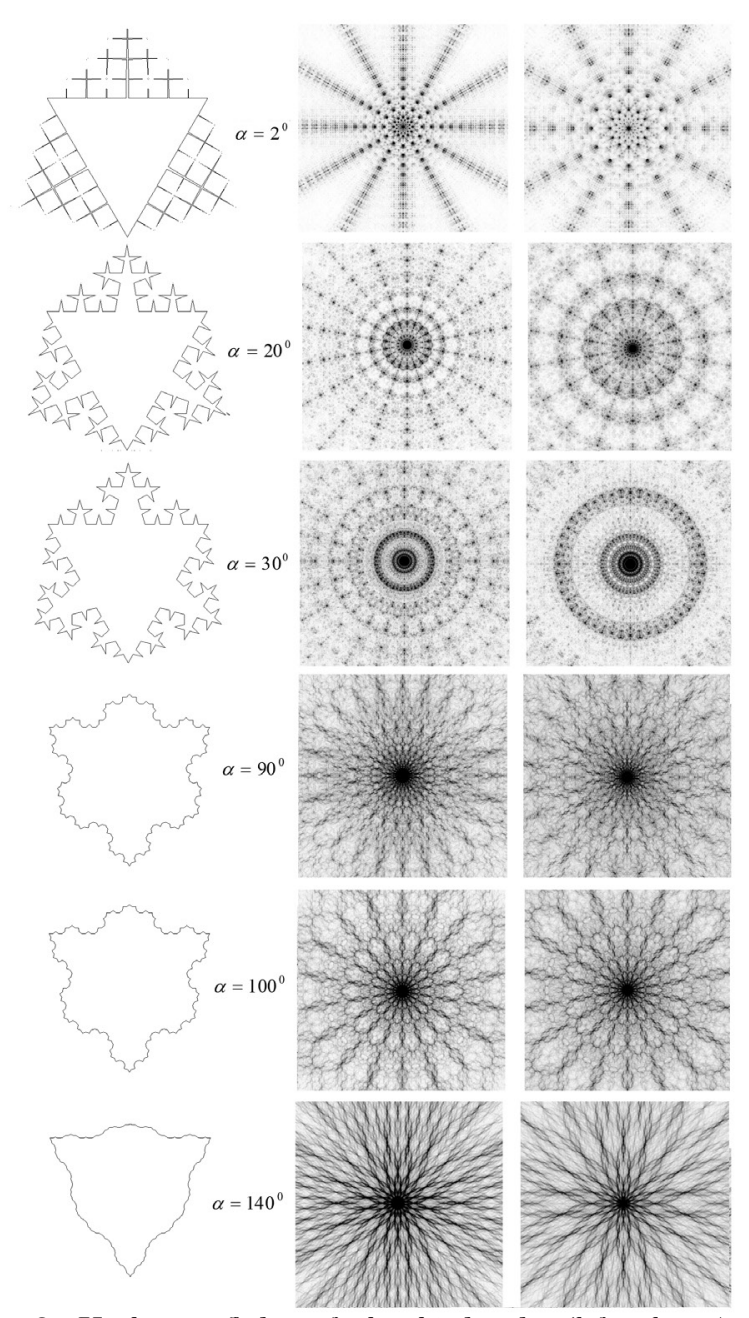

Fig. 8. Koch snowflakes of the third order (left column) with the angles $\alpha=2^{\circ}, 20^{\circ}, 30^{\circ}, 90^{\circ}, 100^{\circ}, 140^{\circ}$ and their Fourier images in two scales (central and right column; small and large plan, respectively).

the 4-th - 6-th generation, in right column magnified in two times central (fractal) part of the Fourier images.

On the contrary, for other values of angle $\alpha$, even slightly different from $60^{\circ}$ (e.g. for $63^{\circ}$ ), diffraction patterns become blurred, and this effect increased while moving off the center, creat-ing the illusion of a "spinning wheel" (see Fig. 9).

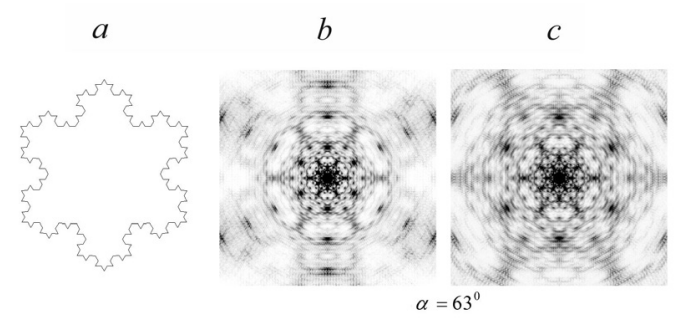

Fig. 9. Koch snowflake of the third order angle (a), its Fourier image (b) and the fractal part of the Fourier image (c). 


\section{PROPERTIES OF NODIFIED KOCH CURVES FOURIER IMAGES}

We also analyzed the properties of the modified Koch curves for which at every stage of construction the generator vertex angle alternately takes on the values $\alpha_{1}$ and $\alpha_{2}$. In Fig. 10 for two cases, when $\alpha_{1}$ and $\alpha_{2}$ equal $57^{\circ}$ and $63^{\circ}-$ (a), and $50^{\circ}$ and $70^{\circ}-(\mathrm{b})$ the Koch curves of the 3 -rd generation (upper row), the Fourier images of the same Koch curves, but of the 6-th generation (middle row), and central parts of them (bottom row) are shown. It is seen that in both cases there is the effect of "rotating wheel", and the blurring of diffraction peaks increases with increasing difference $\alpha_{2}-\alpha_{1}$.

Fig. 11 corresponds to the Fourier images of the 6 -th generation Koch curves, when the values $\alpha_{1}$ and $\alpha_{2}$ are $18^{\circ}$ and $22^{\circ}-(a), 33^{\circ}$ and $39^{\circ}-(b), 60^{\circ}$ and $120^{\circ}(c)$, respectively. In the

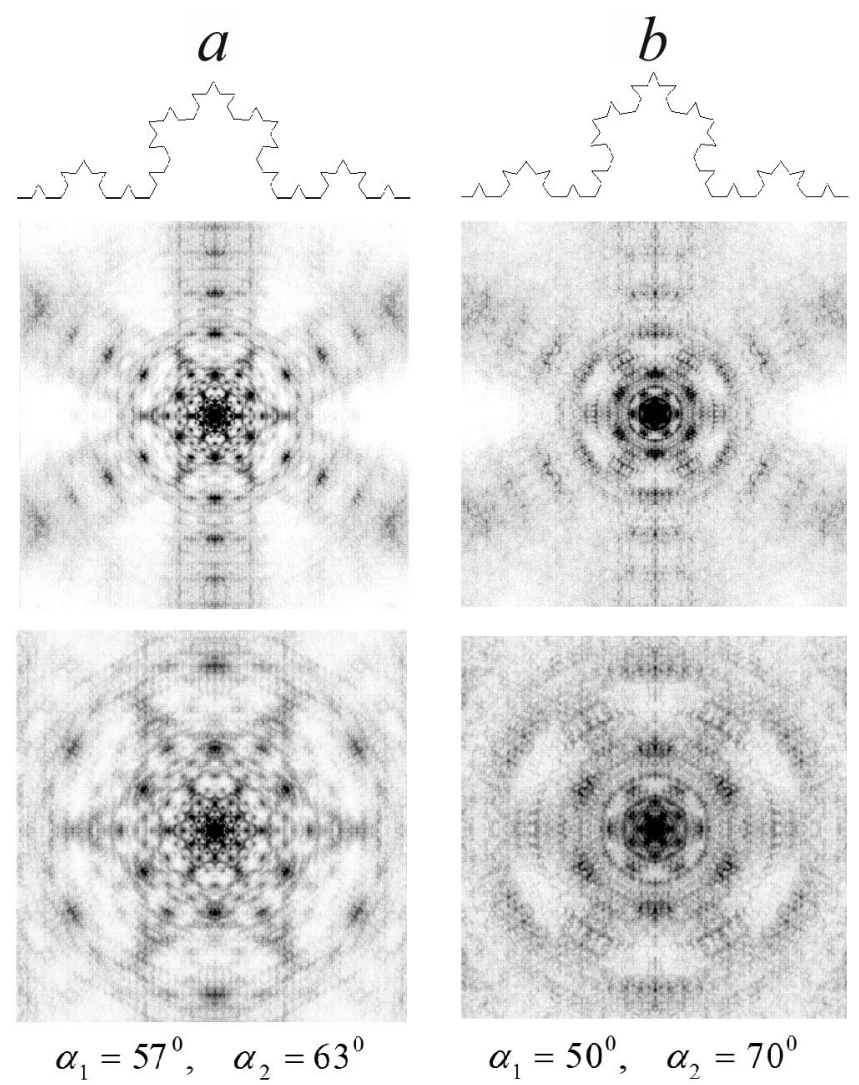

Fig. 10. Modified Koch curves of the 3-rd generation (upper row), the Fourier images of the same Koch curves of the 6-th generation (middle row) and central parts of them (bottom row) for two cases, when at every stage of construction the generator vertex angle alternately takes on the values $\alpha_{1}$ and $\alpha_{2}$ equal $57^{\circ}$ and $63^{\circ}-(a)$, and $50^{\circ}$ and $70^{\circ}-(b)$.

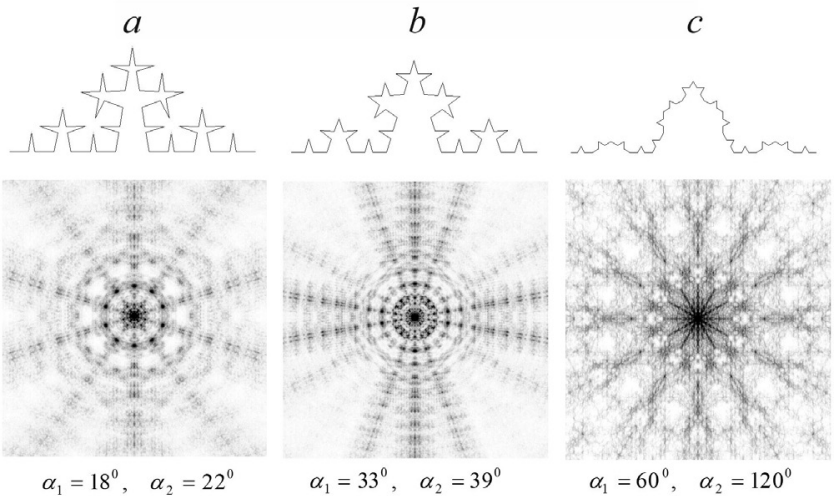

Fig. 11. Fourier images of modified Koch curves for two cases, when at every stage of construction the generator vertex angle alternately takes on the values $\alpha_{1}$ and $\alpha_{2}$ equal $18^{\circ}$ and $22^{\circ}-$ (a), and $60^{\circ}$ and $120^{\circ}-(b)$.

first two cases as in Fig. 10, there is a blurring of diffraction peaks, increasing with distance from the center of the diffraction pattern. In the latter case, the blurring effect is absent.

A diffractograms similar to shown in Fig. 10 and Fig. 11, were used to determine the dimen-sions of the modified Koch curves by methods of circles and of annular zones. The values of the dimension obtained by the first method using dependencies $\bar{I}\left(r_{k}\right)$, is well coincided with the theoretical values determined by formula

$$
D_{f}=\frac{4 \log 2}{2 \log 2+\log \left[\left(1+\sin \left(\alpha_{1} / 2\right)\right)\left(1+\sin \left(\alpha_{2} / 2\right)\right)\right]}=\frac{4 \log 2}{\log m},
$$
where $m=4\left[\left(1+\sin \left(\alpha_{1} / 2\right)\left(1+\sin \left(\alpha_{2} / 2\right)\right]\right.\right.$ is a scale factor. When $\alpha_{1}=\alpha_{2}$, the expression (5) becomes (4). Method of circular areas gave significantly worse results.

\section{CONCLUSION}

Despite the complicated genealogy (see section 1), the fate of fractals Koch has developed extremely well. The total number of dedicated to them theoretical publications is counted by hundreds; the range of practical applications is constantly expanding. Among the latter in the first place should allocate flush-mounted multi-band antenna systems based on generalized and modified Koch fractal, allowing to obtain the same parameters (beam width, sidelobes level, gain and directive gain) as the conventional antenna, but with 
significantly smaller overall dimensions [22-27].

The reduction in size is due to the fact that the length of the generalized Koch prefractal of the $n$-th generation, defined by the expression

$$
L_{n}=\left(\frac{2}{1+\sin (\alpha / 2)}\right)^{n} L_{o}=q^{n} L_{o} \text {, }
$$

where $L_{0}$ is the length of the initiator, corresponding to the size of a conventional vibrator antenna increases with the number of iterations in geometric progression. The denominator of the progression $q$ reaches a maximum equal to 2 at $\alpha \rightarrow 0^{\circ}$, and is drawn into the unit at $\alpha \rightarrow 180^{\circ}$. From (4) and (6) it follows that

$$
L_{n}=\left(\frac{4}{m+2 \sin (\alpha / 2)}\right)^{n} L_{o},
$$

that is, the length of the suggested ceteris paribus increases with lowering of the scale factor or by increasing the fractal dimension.

Antennae using modified Koch curves, when the generator vertex angle $\alpha$ at each stage of construction alternately takes on the values $\alpha_{1}$ and $\alpha_{2}$, also have found application (see eg. [25]).

For the above reasons the results of our research can be useful in determining the real size and capabilities of fractal antennas by diffraction techniques, as well as for the comparison of the di-rectivity diagram with Fourier-images of the corresponding fractals obtained by numerical methods.

\section{REFERENCES}

1. Koch H. von. Sur une courbe continue sans tangente, obtenue par une construction géométrique élémentaire. Arkiv för Matematik, Astronomi och Fysike, 1904, 1:681-704.

2. Takagi T. A simple example of the continuous function without derivative. Proc. Phys.-Math. Japan, 1903, 1:176-177.

3. Hildebrandt TH. A simple continuous function with a finite derivative at no point. Amer. Math. Monthly, 1933, 40(9):547-548.

4. de Rham G. Sur un exemple de fonction continue sans dériveée. Enseign. Math., 1957, 3:71-72.
5. Vepstas L. A gallery of de Rham curves. linas@linas.org http:/ /www.linas.org/math/ de_Rham.pdf, 2006.

6. Arzamastseva GV, Evtikhov MG, Lisovsky FV, Lukashenko LI. Komp'yuternoe modelirovanie difraktsii sveta na fraktal'nykh domennykh strukturakh [Computer simulation of light diffraction on fractal domain structures]. Trudy XIX Mez̧bdunar. sckoly-seminara "Novye magnitnye materialy mikerolektroniki", Moscow, Lomonosov MSU, 2004:632-634 (in Russ.).

7. Arzamastseva GV, Evtikhov MG, Lisovsky FV, Mansvetova EG, Temiryazeva MP. Amorfizatsiya biperiodicheskikh domennykh struktur $\mathrm{v}$ kvaziodnoosnykh magnitnykh plenkakh kriticheskoy tolschiny [Amorphization of periodic domain structures in quasi uniaxial magnetic films of the critical thickness]. ZHETF, 2008, 134(2):282-290 (in Russ.).

8. Arzamastseva GV, Evtikhov MG, Lisovsky FV, Mansvetova EG. Furie obrazy fraktal'nykh obektov [The Fourier images of fractal objects]. Iqvestiya RAN, ser. fir., 2010, 74(10):1430-1432 (in Russ.).

9. Alain C, Cloitre M. Optical diffraction on fractals. Phys. Rev. B, 1986, 33(5):3566-3569.

10. Sakurada Y., Uozumi J., Asakura T. Diffraction fields of fractally bounded apertures. Optical review, 1994, 1(1):3-7.

11. Uozumi J, Kimura H, Asakura T. Fraunhofer diffraction by Koch fractals. J. Mod. Optics, 1990, 37(6):1011-1031.

12. Zinchik AA, Muzychenko YaB, Stafeev SK. O printsipakh amplitudnoy i amplitudnofazovoy prostranstvennoy fil'tratsii [On the principles of the amplitude and phaseamplitude spatial filtering]. Izv. VUZov. Priborostroenie, 2007, 50(7):46-52 (in Russ.).

13. Zinchik AA, Muzychenko YaB, Smirnov AV, Stafeev SK. Raschet fraktal'noy razmernosti regulyarnykh fraktalov po kartine difraktsii $\mathrm{v}$ dal'ney zone [Calculation of fractal dimension of the regular fractals on the diffraction 
pattern in the far zone]. Nauchno-tekhnichesky vestnik SPbGU ITMO, 2009, 2(60):17-24 (in Russ.).

14. Mandelbrot BB. The fractal geometry of nature. New York, WH Freeman, 1983.

15. Pietronero L, Tosatti E (eds.) Fractals in physics, North-Holland, Amsterdam, 1986.

16. Feder J. Fractals. N.Y.-London, Plenum Press, 1988.

17. Schroeder M. Fractals, chaos, power laws. Minutes from an infinite paradise. N.Y., WH Freeman, 1992.

18. Stoyan D, Stoyan H. Fractals, random shapes and point fields. Chichester, John Wiley and Sons, 1994, 399 p.

19. Peitgen H-O, Jürgens H, Saupe D. Chaos and fractals. New frontiers of science. New York, Springer-Verlag, 2004, 864 p.

20. Gouyet J-F. Physics and fractal structures. Paris, Ecole Polytechnique, 1995, 247 p.

21. Arzamastseva GV, Evtikhov MG, Lisovsky FV, Mansvetova EG. Komp'yuternoe modelirovanie difraktsii Fraungofera na H-fraktalakh i krivykh Peano [Computer simulation of Fraunhofer diffraction at the $\mathrm{H}$-fractals and Peano curves]. Elektromagnitnye volny $i$ elektronnye sistemy, 2012, 7:8-58 (in Russ.).

22. Cohen N. Fractal antennas: Part 1. Communications Quarterly, Summer 1995:7-22.

23. Cohen N. Self-similarity and the geometric requirements for frequency independence in antennae. Fractals, 1999, 7(1):79-84.

24. Best SR. The Koch fractal monopole antenna: The significance of fractal geometry in determin-ing antenna performance. Proceedings of the 2001 Antenna Applications Symposium. Allerton Park Monticello, Illinois, 2000.-www.ecs.umass.edu/ece/allerton/ papers2001/2001-p194.pdf.

25. Vinoy KJ, Abraham JK, Varadan VK. On the relationship between fractal dimension and the performance of multi-resonant dipole antennas using Koch curves. IEEE
Transactions on Antennas and Propagation, 2003, 51(9):2296-2303.

26. Karaboikis M, Soras C, Tsachtsiris G, Makios $\mathrm{V}$. Four-element printed monopole antenna systems for diversity and MIMO terminal devices. Proceedings of the 17th International Conference on Applied Electromagnetics and Communications, Dubrovnik, 2003:193-196.

27. Tsachtsiris G, Soras C, Karaboikis M, Makios V. A printed folded Koch monopole antenna for wireless devices. Microwave and Optical Technology Letters, 2004, 40(5):374-378. 